\title{
Eficácia do Diquat No ConTROLE dE Hydrilla verticillata, Egeria densa E Egeria najas E TOXICIDADE AGUDA PARA O GUARU (Phallocerus caudimaculatus), EM CONDIÇõES DE LABORATÓRIO' ${ }^{1}$
}

\author{
Efficacy of Diquat in the Control of Hydrylla verticillata, Egeria densa and Egeria najas and \\ its Acute Toxicity to Phallocerus caudimaculatus, under Laboratory Conditions
}

\author{
HENARES, M.N.P. ${ }^{2}$, REZENDE, F.R.L. ${ }^{3}$, GOMES, G.R. ${ }^{3}$, CRUZ, C. ${ }^{3}$ e PITELLI, R.A. ${ }^{3}$
}

\begin{abstract}
RESUMO - No Brasil, as macrófitas aquáticas submersas, Egeria densa e Egeria najas, têm causado prejuízos aos usos múltiplos da água. Hydrilla verticillata foi recentemente introduzida, mas tem histórico como planta problemática nos EUA, no México e na Austrália. O objetivo deste trabalho foi avaliar as suscetibilidades relativas dessas três macrófitas aquáticas ao diquat e os riscos da utilização desse herbicida para o guaru (Phallocerus caudimaculatus). Para isso, foram instalados ensaios em condições de laboratório, a fim de avaliar a suscetibilidade relativa das três macrófitas por meio da manutenção de ponteiros dessas plantas em soluções contendo 0,0; 0,2; 0,4; 0,8; e 1,6 $\mathrm{mg} \mathrm{L}^{-1}$ de diquat (Reward ${ }^{\circledR}$ ) por 14 dias. A avaliação foi realizada pela variação do acúmulo de matéria fresca e do comprimento dos ponteiros no periodo de exposição ao herbicida. $H$. verticillata mostrou maior sensibilidade ao diquat em comparação com as duas macrófitas do gênero Egeria, mesmo em baixas concentrações do herbicida. Nas maiores concentrações, $E$. densa mostrou maior sensibilidade que $E$. najas. O risco da aplicação do diquat para $P$. caudimaculatus foi estimado pela toxicidade aguda. Alevinos de P. caudimaculatus de 0,4 $\pm 0,2 \mathrm{mg}$ foram expostos a soluções de 0,0; 1,0; 5,$0 ; 10,0 ; 15,0 ; 20,0 ; 25,0 ;$ e 30,0 $\mathrm{mg} \mathrm{L}^{-1}$ de diquat. A concentração letal de 50\% (CL(I) $\left.(50 ; 96 \mathrm{~h})\right)$ do diquat estimada para $P$. caudimaculatus foi de $7,17 \mathrm{mg} \mathrm{L}^{-1}$. Para $P$. caudimaculatus, a toxicidade aguda foi superior à concentração recomendada para o controle de macrófitas aquáticas submersas, indicando risco muito baixo para esse peixe.
\end{abstract}

Palavras-chave: macrófitas aquáticas, toxicidade, herbicida, risco ambiental.

\begin{abstract}
In Brazil, the submerged plants Egeria densa and Egeria najas have caused damage to multiple uses of water. Hydrilla verticillata has been recently introduced, but it has a history as a problem plant in the U.S., Mexico and Australia. The objectives of this work were to assess the relative susceptibilities of these three macrophytes to diquat and the risks of using this herbicide for fish guppy (Phallocerus caudimaculatus). Thus, laboratory assays were set up to assess the relative susceptibility of the three macrophytes. Pointers of these plants were kept in solutions containing 0.0, 0.2, 0.4, 0.8 and $1.6 \mathrm{mg} \mathrm{L}^{-1}$ diquat (Reward ${ }^{\circledR}$ ) for 14 days. The evaluation was performed based on the variation of fresh matter weight and length of the pointers during the period of exposure to the herbicide. $\boldsymbol{H}$. verticillata was more sensitive to diquat, compared with the two Egeria macrophytes, even at low herbicide concentrations. At higher herbicide concentrations, $\boldsymbol{E}$. densa was more sensitive than $\boldsymbol{E}$. najas. The risk of applying diquat to $\boldsymbol{P}$. caudimaculatus was estimated by acute toxicity. Fingerlings of $\boldsymbol{P}$. caudimaculatus of $0.4 \pm 0.2 \mathrm{~g}$ were exposed to solutions of 0.0, 1.0, 5.0, 10.0, 15.0, 20.0, 25.0 and $30.0 \mathrm{mg} \mathrm{L^{-1 }}$ of diquat. The $50 \%$ lethal concentration (LC (I) (50.96h)) of diquat estimated for P. caudimaculatus was $7.17 \mathrm{mg} \mathrm{L}^{-1}$. For $\boldsymbol{P}$. caudimaculatus, acute toxicity was higher than the concentration recommended for the control of submerged plants, indicating very low risk for this fish.
\end{abstract}

Keywords: aquatic macrophytes, toxicity, herbicide, environmental risk.

1 Recebido para publicação em 5.2.2011 e na forma revisada em 6.5.2011

2 Centro de Aquicultura , Universidade Estadual Paulista "Júlio de Mesquita Filho" - UNESP, 14884-900 Jaboticabal-SP, $<$ henaresmnp@yahoo.com.br>; ${ }^{3}$ Núcleo de Estudos e Pesquisas Ambientais em Matologia - NEPEAM, UNESP. 


\section{INTRODUÇÃO}

As alterações antrópicas nos corpos d'água têm favorecido o desenvolvimento de populações de determinadas plantas aquáticas, o que pode causar prejuízos aos usos múltiplos da água e do corpo hídrico (Pitelli et al., 2008). Em ambientes lênticos, com água transparente, sedimento rico, $\mathrm{pH}$ neutro ou levemente alcalino, as macrófitas submersas têm sido favorecidas (Martins, 2005). Nessas condições, Egeria densa e Egeria najas, nativas da América do sul, e Hydrilla verticillata, proveniente da Ásia, todas pertencentes à família Hydrocharitaceae, causam sérios problemas em corpos hídricos no Brasil, como na Usina Hidrelétrica (UHE) Souza Dias, associada ao reservatório de Jupiá, SP (Marcondes et al., 2003), UHE de Fontes, associada aos complexos de reservatórios de Santana e Vigários, RJ (Pitelli et al., 2008), e UHE Paulo Afonso, PE (Nascimento et al., 1998). No caso específico de Jupiá, as macrófitas aquáticas submersas causam grandes prejuízos para a geração de energia elétrica, a pesca esportiva e profissional e os esportes náuticos (Mustafá et al., 2010), bem como à fauna aquática, pela dificuldade de locomoção de peixes de grande porte e anóxia noturna (Carnwall et al., 2001). Nessas condições, o controle dessas plantas daninhas aquáticas torna-se fundamental para manutenção da função social, econômica e ambiental do corpo hídrico (Pitelli et al., 2008).

Entre as opções de manejo dessas plantas, o controle químico tem sido empregado em vários países, pois promove resultado rápido e eficiente, sendo seguro para a vida animal quando utilizado dentro das recomendações técnicas. O controle mecânico, por exemplo, promove o risco de coleta indevida de animais jovens e proporciona a dispersão de plantas com reprodução vegetativa (Cardoso et al., 2003 e Mustafá et al., 2010).

No Brasil há apenas um herbicida registrado para o controle de plantas daninhas aquáticas; trata-se do fluridone, que é específico para macrófitas submersas (Pitelli et al., 2008). Vários outros herbicidas têm sido usados em outras regiões do mundo e poderiam ser utilizados no território nacional, porém a falta de estudos básicos sobre a ação deles no controle das espécies-alvo e os seus efeitos sobre os organismos não alvos ainda não foi considerada suficiente para que as agências regulatórias pudessem autorizar sua utilização.

O herbicida diquat (1-1'-etileno-2-2'dibrometo de bipiridílio) é considerado um herbicida eficiente no controle das duas macrófitas submersas do gênero Egeria, segundo estudos realizados no Brasil em condições de mesocosmos (Martins et al., 2005, 2007, 2008) e de campo (Velini, 2005). Nos EUA, ele tem sido utilizado no controle de $E$. densa e de $H$. verticillata e age na formação de radicais livres a partir de elétrons capturados do fotossistema I. Esses radicais promovem a oxidação de lipídios, que tem como principal consequência a ruptura de membranas, levando à necrose de tecidos (Dodge, 1991; Hess, 1993).

Considerando a possibilidade de utilização do diquat para o controle de $H$. verticillata, $E$. densa e E. najas em lagos e, principalmente, em reservatórios de hidrelétricas do Brasil, este trabalho foi conduzido em condições de laboratório e teve como principais objetivos realizar uma avaliação comparativa das suscetibilidades das três principais macrófitas submersas que causam problemas em reservatórios e estimar o valor CL(I)(50;96h) desse herbicida para o guaru (Phallocerus caudimaculatus), uma espécie cosmopolita pertencente à familia Poeciliidae que pode ser utilizada como organismo-teste e bioindicador das concentrações de produtos químicos nos ambientes aquáticos. Este trabalho não pôde ser realizado em campo, devido à ausência de registro no Brasil; o trabalho de Velini (2005) foi realizado com registro especial temporário do IBAMA.

\section{MATERIAL E MÉTODOS}

Os experimentos foram conduzidos no Núcleo de Estudos e Pesquisas Ambientais em Matologia (Nepeam) da Faculdade de Ciências Agrárias e Veterinárias, UNESP, Jaboticabal.

Para determinação da suscetibilidade das três macrófitas ao diquat, o experimento foi conduzido em sala climatizada mantida na temperatura de $25 \pm 2{ }^{\circ} \mathrm{C}$ e fotoperíodo de 12 horas. O produto comercial utilizado foi o Reward $^{\circledR}$, específico para ambientes aquáticos, 
na concentração de $447 \mathrm{~g} \mathrm{~L}^{-1}$ do sal de dibrometo de diquat ou $240 \mathrm{~g} \mathrm{~L}^{-1}$ do cátion diquat.

Em frascos plásticos transparentes com capacidade para $1,7 \mathrm{~L}$, contendo $270 \mathrm{~g}$ de areia como sedimento e 1,3 L de água, foram transplantados tufos de três ponteiros, com $10 \mathrm{~cm}$ de comprimento cada.

Os ponteiros foram coletados em plantas que apresentavam bom estado sanitário e nutricional e tiveram sua biomassa fresca avaliada em balança de precisão (Marte ${ }^{\circledR}$ modelo AS2000C), antes da colocação nos frascos. Antes da determinação da biomassa fresca foi retirado o excesso de água dos ponteiros, pressionando-os gentilmente contra papel-chupão.

O experimento foi instalado no delineamento experimental inteiramente casualizado com cinco repetições, e os tratamentos foram dispostos em esquema fatorial $3 \times 5$, tendo como variáveis três espécies de macrófitas (E. densa, E. najas e $H$. verticillata) e cinco concentrações de diquat $(0,0 ; 0,2 ; 0,4 ; 0,8$; e $\left.1,6 \mathrm{mg} \mathrm{L}^{-1}\right)$. Esse herbicida foi colocado nos frascos após três dias do transplante dos ponteiros.

O período de exposição das macrófitas ao herbicida foi de 14 dias. Ao final desse período, os ponteiros foram avaliados quanto ao comprimento e à biomassa fresca. O efeito de controle do diquat sobre o crescimento e acúmulo de biomassa nos ponteiros foi determinado pela equação proposta por Henderson \& Tilton (1967).

Quanto à análise estatística dos dados do peso e do comprimento dos ponteiros, foi realizada a análise de variância (ANOVA), com as médias comparadas pelo teste de Tukey a $5 \%$, utilizando-se o software $\mathrm{SAS}^{\circledR}$ Inst. (SAS, 1998).

Para avaliação da toxicidade aguda do diquat ao $P$. caudimaculatus, foram utilizados peixes com peso de $0,4 \pm 0,2$ gramas, mantendo-se a densidade dos aquários em $1 \pm 0,2 \mathrm{~g} \mathrm{~L}^{-1}$, de acordo com a recomendação da Cetesb (1999). Os peixes foram aclimatados por 10 dias na sala de bioensaios, com temperatura de $27 \pm 2{ }^{\circ} \mathrm{C}$ e fotoperíodo de 12 horas. A aclimatação foi realizada em uma caixa com capacidade para $250 \mathrm{~L}$, com sistema de aeração contínuo, promovido por bombas de ar e circulação contínua de água. Nesse período, os peixes foram alimentados com ração comercial, ad libitum, uma vez ao dia. A água utilizada nos testes de toxicidade apresentou as seguintes características fisico-químicas: temperatura: $26,85 \pm 0,6{ }^{\circ} \mathrm{C} ; \mathrm{pH}: 7,54 \pm 0,5$; oxigênio dissolvido: $7,59 \pm 0,6 \mathrm{mg} \mathrm{L}^{-1}$; condutividade elétrica: $0,180 \mu \mathrm{S} \mathrm{cm}^{-1}$; e dureza: $58 \pm 1,9 \mathrm{mg}$ de $\mathrm{CaCO}_{3} \mathrm{~L}^{-1}$.

No que se refere ao controle de sensibilidade dos organismos-teste, foram realizados testes periódicos de toxicidade aguda, com 96 horas de duração, utilizando como substância de referência o dicromato de potássio com teor de pureza de $99 \%$, de acordo com a recomendação do Ibama (1987). A concentração letal (CL(I)(50;96h)) do dicromato de potássio estimada para $P$. caudimaculatus foi de $128,61 \mathrm{mg} \mathrm{L}^{-1}$, estando de acordo com as recomendações de sensibilidade para essa espécie (Cruz et al., 2008).

Para a realização dos testes preliminares e de determinação da toxicidade aguda do diquat para o guaru, os aquários foram dispostos em delineamento experimental inteiramente casualizado, com três repetições e três animais por repetição, sendo o período de exposição dos peixes de 96 horas em sistema estático, sem substituição ou sifonagem de água e sem alimentação durante o período.

Os testes preliminares de toxicidade aguda foram realizados utilizando-se seis concentrações de diquat e um tratamento controle (Rand \& Petrocelli 1985). Nesses testes foram determinados os intervalos de concentração de diquat entre os valores que causaram 0 e $100 \%$ de mortalidade dos peixes, para serem utilizados nos testes definitivos, segundo as recomendações da Cetesb (1999). Nos testes de determinação da toxicidade aguda (CL(I) $(50 ; 96 \mathrm{~h})$ ), os peixes foram expostos às concentrações de 0,$0 ; 1,0 ; 5,0 ; 10,0 ; 15,0$; 20,$0 ; 25,0$; e $30,0 \mathrm{mg} \mathrm{L}^{-1}$ e os valores de CL(I)(50;96h) foram calculados pelo método Trimmed Spearman-Karber (Hamilton et al., 1977).

\section{RESULTADOS E DISCUSSÃO}

Após 14 dias de exposição, para $H$. verticillata e $E$. densa, os pesos da matéria 
fresca dos ponteiros que tiveram contato com o diquat foram significativamente inferiores ao da testemunha. Para $H$. verticillata e E. densa não houve diferença estatística entre os ponteiros expostos às diferentes concentrações avaliadas. Para E. najas ocorreu similaridade estatística nas biomassas dos ponteiros que cresceram na testemunha e na concentração de $0,2 \mathrm{mg} \mathrm{L}^{-1}$, que foram superiores aos valores observados nas concentrações de 0,4 a 1,6 $\mathrm{mg} \mathrm{L}^{1}$ (Tabela 1).

A equação de Henderson \& Tilton (1967) considera as diferenças de valores da testemunha e do tratamento com o diquat no periodo avaliado (14 dias), contemplando o potencial de crescimento da planta no período e o crescimento ocorrido no tratamento em avaliação. Assim, para $H$. verticillata as reduções de biomassa variaram entre 71,2 e $78,6 \%$; para E. densa, entre 42,7 e $52,0 \%$; e para E. najas, entre 18,7 e $34,7 \%$ (Figura 1).

$\mathrm{Na}$ análise das variações de comprimento dos ponteiros (Tabela 1) apenas para $H$. verticillata, os valores observados na testemunha foram estatisticamente superiores aos dos ponteiros expostos às quatro concentrações avaliadas. Pela equação de Henderson $\&$ Tilton (1967), os valores de controle variaram de 89,5 a $93,5 \%$ (Figura 2). Para E. densa, esses valores variaram entre 26,8 e $69,6 \%$. Considerando os comprimentos dos ponteiros após 14 dias de exposição ao diquat, houve similaridade estatística entre os valores da testemunha e das concentrações de 0,2 e $0,4 \mathrm{mg} \mathrm{L}^{-1}$, os quais superaram os observados para 0,8 e $1,6 \mathrm{mg} \mathrm{L}^{-1}$. Em E. najas, os valores de controle variaram entre 28 e $37 \%$ (Figura 2).

Na Tabela 1, é possível observar que na testemunha não ocorreu diferença no acúmulo de matéria fresca entre as três macrófitas, mas $H$. verticillata apresentou maior elongação do ponteiro que as duas plantas do gênero Egeria. Quando em contato com o diquat, os valores de acúmulo de matéria fresca para $H$. verticillata foram menores que para as outras duas macrófitas, com exceção de $E$. densa exposta à concentração de $0,2 \mathrm{mg} \mathrm{L}^{-1}$. A elongação do ponteiro em $H$. verticillata foi muito mais inibida pelo diquat em relação às outras duas espécies nas doses mais baixas $\left(0,2\right.$ e $\left.0,4 \mathrm{mg} \mathrm{L}^{-1}\right)$. Nas duas concentrações mais elevadas, a elongação de $E$. densa também foi bastante prejudicada pelo herbicida, e o seu comprimento foi estatisticamente inferior ao de E. najas.

Esse tipo de bioensaio é importante na fase anterior à liberação comercial de um herbicida e deve ser realizado na fase de registro especial temporário 01 , pois fornece evidência expressiva da suscetibilidade relativa das plantas daninhas que serão alvos de controle. Os resultados mostraram que $H$. verticillata mostrou maior sensibilidade ao diquat quando comparado com as duas macrófitas do gênero Egeria, mesmo em concentração baixa do herbicida.

Tabela 1 - Médias dos valores do peso da matéria fresca e do comprimento dos ponteiros das três macrófitas submersas, após 14 dias de exposição em concentrações crescentes de diquat, e os respectivos resultados dos desdobramentos dos graus de liberdade dos tratamentos e os resultados do teste de Tukey $(5 \%)$

\begin{tabular}{|c|c|c|c|c|c|}
\hline \multirow{3}{*}{ Macrófita } & \multicolumn{5}{|c|}{ Concentração de diquat $\left(\mathrm{mg} \mathrm{L}^{-1}\right)$} \\
\hline & \multicolumn{5}{|c|}{ Peso $(\mathrm{g})$} \\
\hline & 0,0 & 0,2 & 0,4 & 0,8 & 1,6 \\
\hline H. verticillata $(\mathrm{g})$ & $3,10 \mathrm{Aa}^{\frac{1}{1}}$ & $0,84 \mathrm{Bc}$ & $0,62 \mathrm{Bb}$ & $0,77 \mathrm{Bb}$ & $0,76 \mathrm{Bb}$ \\
\hline E. densa $(\mathrm{g})$ & $2,99 \mathrm{Aa}$ & $1,64 \mathrm{Bb}$ & $1,57 \mathrm{Ba}$ & $1,45 \mathrm{Ba}$ & $2,15 \mathrm{Ba}$ \\
\hline \multirow[t]{2}{*}{ E. najas $(\mathrm{g})$} & $3,42 \mathrm{Aa}$ & $2,74 \mathrm{Aa}$ & $1,85 \mathrm{Ba}$ & $2,05 \mathrm{Ba}$ & $1,95 \mathrm{Ba}$ \\
\hline & \multicolumn{5}{|c|}{ Comprimento $(\mathrm{cm})$} \\
\hline H. verticillata $(\mathrm{cm})$ & $76,4 \mathrm{Aa}$ & $8,0 \mathrm{Bb}$ & $7,2 \mathrm{Bb}$ & $5,0 \mathrm{Bb}$ & $6,0 \mathrm{Bb}$ \\
\hline E. densa $(\mathrm{cm})$ & $39,5 \mathrm{Ab}$ & $28,9 \mathrm{Aba}$ & $24,2 \mathrm{Aba}$ & $6,0 \mathrm{Cb}$ & $18,0 \mathrm{BCb}$ \\
\hline E. najas $(\mathrm{cm})$ & $49,2 \mathrm{Ab}$ & $35,4 \mathrm{Aba}$ & $32,8 \mathrm{Ba}$ & $31,0 \mathrm{Ba}$ & $33,0 \mathrm{~A} \mathrm{Ba}$ \\
\hline
\end{tabular}

1/ Médias acompanhadas de mesma letra não diferem estatisticamente entre si (Tukey 5\%). Letras maiúsculas comparam os efeitos das concentrações de diquat; letras minúsculas comparam as macrófitas avaliadas. 


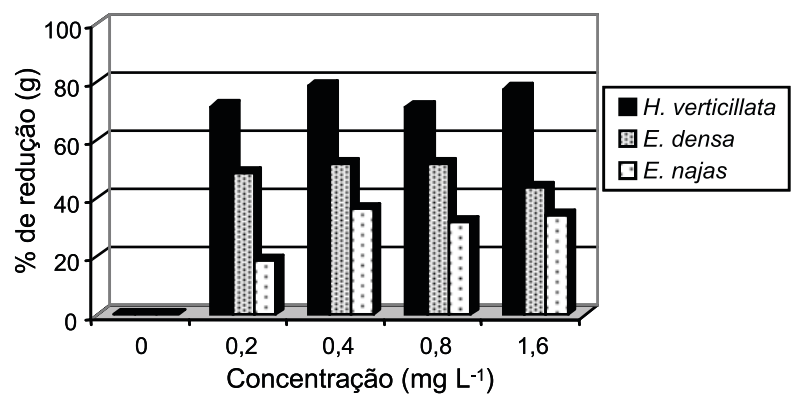

Figura 1 - Representação gráfica das porcentagens de redução da massa das macrófitas aquáticas após 14 dias de exposição às doses crescentes de diquat (Henderson \& Tilton, 1976).

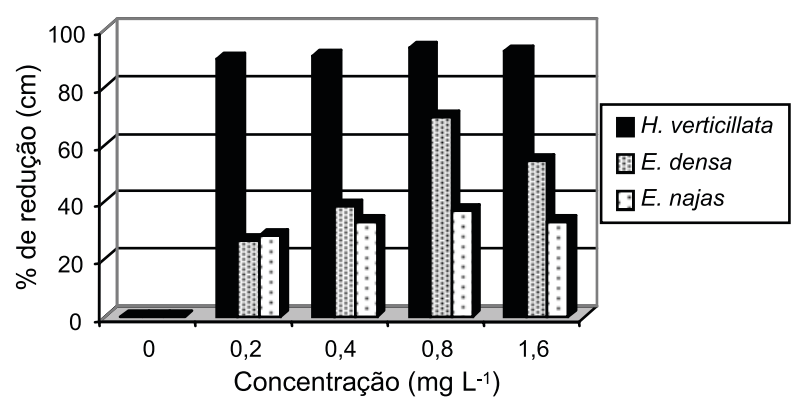

Figura 2 - Representação gráfica das porcentagens de redução do comprimento das macrófitas aquáticas após 14 dias de exposição às doses crescentes de diquat (Henderson \& Tilton, 1976).

Em concentrações maiores, E. densa mostrou maior sensibilidade que E. najas. No entanto, é importante considerar que, além da diferença entre as espécies, há expressiva diferença entre acessos de Egeria no Brasil para sensibilidade aos herbicidas diquat e fluridone (Martins et al., 2007) e de $H$. verticillata nos EUA para fluridone (Netherlands et al., 2000).

Em condições de mesocosmos, Martins et al. (2005) não observaram controle de $E$. densa e E. najas nas concentrações de 0,1 e $0,25 \mathrm{mg} \mathrm{L}^{-1} \mathrm{em}$ avaliação realizada aos 14 dias de aplicação. Contudo, nas concentrações de 0,5, 1,0 e 1,5 $\mathrm{mg} \mathrm{L}^{-1}$, o herbicida proporcionou 95, 96 e $96 \%$ de controle, respectivamente. Aos 26 dias essas concentrações apresentaram $98 \%$ de controle de E. najas e $100 \%$ de controle de E. densa, evidenciando mais uma vez que a primeira é mais tolerante ao diquat e também apresentou maior intensidade de rebrota. Para o controle de E. najas aos 7 dias, foi necessário um período de exposição de $120 \mathrm{~min}$ a $0,075 \mathrm{mg} \mathrm{L}^{-1}$ (Martins et al., 2008).

No Brasil, $H$. verticillata é uma planta exótica invasora de introdução recente, para a qual não há estudos de controle publicados. Nos EUA, Blackburn \& Weldon (1970) e Gangstad (1978) constataram eficácia do diquat no controle de $H$. verticillata nas concentrações iguais ou acima de $0,25 \mathrm{mg} \mathrm{L}^{-1}$. Todavia, quando esse herbicida foi associado ao sulfato de cobre, ele foi eficaz apenas em concentrações iguais ou superiores a $1,0 \mathrm{mg} \mathrm{L}^{-1}$.

A concentração letal de 50\% (CL(I) (50;96h)) do diquat estimada para o guaru (P. caudimaculatus) foi de $7,17 \mathrm{mg} \mathrm{L}^{-1}$, com limite inferior de $4,43 \mathrm{mg} \mathrm{L}^{-1}$ e superior de $10,90 \mathrm{mg} \mathrm{L}^{-1}$. Na Figura 3 são apresentados a relação concentração-resposta e o $\mathrm{R}^{2}$ ajustado pela equação linear (Hamilton et al., 1977).

Nos tratamentos controle e $1,0 \mathrm{mg} \mathrm{L}^{-1}$ não ocorreu mortalidade dos peixes expostos. No tratamento com $5,0 \mathrm{mg} \mathrm{L}^{-1}$ houve $40 \%$ de mortalidade; com $10 \mathrm{mg} \mathrm{L}^{-1}$, 50\%; com 15 e $20 \mathrm{mg} \mathrm{L}^{-1}, 70 \%$; com $25 \mathrm{mg} \mathrm{L}^{-1}, 90 \%$; e com $30 \mathrm{mg} \mathrm{L}^{-1}, 100 \%$ de mortalidade dos peixes expostos.

Quando comparado com os resultados ora obtidos, o diquat foi mais tóxico para o guaru do que para Carassius auratus com CL(50;96h)

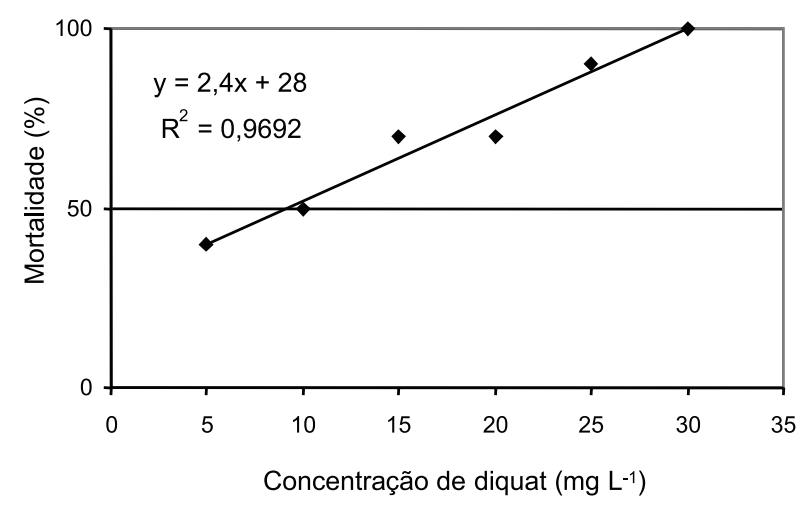

Figura 3 - Representação gráfica dos valores de mortalidade de Phallocerus caudimaculatus em várias concentrações do diquat e da equação de correlação concentração-mortalidade do peixe. 
de 85,0 $\mathrm{mg} \mathrm{L}^{-1}$ (Berry Jr., 1984); carpa-capim (Ctenopharyngodon idella) com $53,0 \mathrm{mg} \mathrm{L}^{-1}$ (E1 Denn et al., 1992); L. macrocephalus com 34,76 $\mathrm{mg} \mathrm{L}^{-1}$ (Henares et al., 2007); e O. niloticus com $37,28 \mathrm{mg} \mathrm{L}^{-1}$ (Henares et al., 2008). O diquat foi menos tóxico para Stizostesdion viterum, Micropterus salmonides e Micropterus dolomieu, com CL(50;96h) entre 0,74 e 4,9 $\mathrm{mg} \mathrm{L}^{-1}$ (Paul et al., 1994).

$\mathrm{O}$ diquat apresentou excelente potencial de controle das três macrófitas aquáticas submersas avaliadas, com melhores perspectivas para $H$. verticillata, E. densa e E. najas, em ordem decrescente. A toxicidade aguda para o guaru foi muito superior à concentração recomendada para o controle de macrófitas aquáticas nos EUA (Blackburn \& Weldon, 1970) e naquelas que proporcionaram controle satisfatório no trabalho de Martins et al. (2007), indicando muito baixo risco para esse peixe. É importante observar que na literatura citada também os valores de CL(I)(50;96h) foram superiores às concentrações praticadas para o controle dessas macrófitas submersas.

Pela classificação proposta por Zucker (1985), o diquat apresenta risco moderado, o que indica continuidade dos estudos ecotoxicológicos, nos quais o comportamento ambiental do diquat deve ser aferido, especialmente para organismos nativos de corpos hídricos brasileiros.

\section{LITERATURA CITADA}

BERRY Jr., C. R. Toxicity of the herbicides diquat and endothal to goldfish. Environ. Pollut., v. 34, n. 3, p. 251-258, 1984.

BLACKBURN, R. D.; WELDON, L. W. Control of Hydrilla verticillata. Hyacinth Control J., v. 8, n. 1, p. 4-9, 1970.

CARDOSO, L. R. et al. Sensibilidade a herbicidas de acessos de aguapé coletados em reservatórios do Estado de São Paulo. Planta Daninha, v. 21, n. 1, p. 61-67, 2003.

CARNWALL, M. et al. Avaliação do impacto da colonização de Egeria densa sobre a variação dos teores de oxigênio dissolvido na água e sobrevivência de Poecillia reticulata. Ciência das Plantas Daninhas, v. 7, n. 1, p. 9-13, 2001.

\section{COMPANHIA DE TECNOLOGIA DE SANEAMENTO} AMBIENTAL - CETESB. Métodos de avaliação da toxicidade de poluentes a organismos aquáticos. Água teste de toxicidade aguda com peixes - parte I - sistema estático. São Paulo: 1999. v. 2. p. 1-29.
CRUZ, C. et al. Sensibilidade de peixes neotropicais ao dicromato de potássio. J. Braz. Soc. Ecotoxicol., v. 3, n. 1, p. $53-55,2008$,

DODGE, A. D. Photosyntehesis. In: KIKWOOD, R. C. Target sites of herbicide action. Glasgow: University of Stranthclyde, 1991. p. 1-56.

EL DEEN, M. A. S. et al. Acute toxicity and some hematological changes in grass carp exposed to diquat. J. Aquatic Animal Health, v. 4, n. 4, p. 277-280, 1992.

GANGSTAD, E. O. Chemical control Hydrilla. J. Aquatic Plant Manag., v. 16, n. 1, p. 38-40, 1978.

HAMILTON, M. A. et al. Trimmed Spearman-Karber method for estimating median lethal concentrations in toxicity bioassays. Environ. Sci. Technol., v. 7, n. 2, p. 714-719, 1977.

HENARES, M. N. P. et al. Toxicidade aguda e efeitos histopatológicos do diquate na brânquia e no fígado do piauçu (Leporinus macrocephalus). Pesticidas: Ecotoxicol.

Meio Amb., v. 17, p. 107-116, 2007.

HENARES, M. N. P. et al. Toxicidade aguda e efeitos histopatológicos do herbicida diquat na brânquia e no fígado da tilápia nilótica (Oreochromis niloticus). Acta Sci. Biol. Sci., v. 30, n. 1, p. 77-82, 2008.

HENDERSON, C. F.; TILTON, E. W. Tests with acaricides against the brow wheat mite. J. Econ. Entomol., v. 48, n. 2, p. $157-161,1955$.

HESS, F. D. Herbicides effects on plant structure, physiology, and biochemistry. In: ALTMAN, J. Pesticide interactions in crop production beneficial and deleterious effects. London: CRC Press, 1993 p. $123-165$.

INSTITUTO BRASILEIRO DO MEIO AMBIENTE E DOS RECURSOS NATURAIS RENOVÁVEIS - IBAMA. Avaliação da toxicidade aguda para peixes. In: Manual de testes para avaliação de ecotoxicidade de agentes químicos. Brasília: 1987. Parte D. 3.

MARCONDES, D. A. S. et al. Eficiência de fluridone no controle de plantas daninhas aquáticas submersas e efeito sobre algumas características ambientais. Planta Daninha, v. 20 , p. $63-73,2003$.

MARTINS, D. et al. Controle de Egeria densa e Egeria najas em caixa d' água utilizando o herbicida diquat.

Planta Daninha, v. 23, n. 2, p. 381-385. 2005.

MARTINS, D. et al. Sensibilidade de diferentes acessos de Egeria najas e Egeria densa aos herbicidas diquat e fluridone. Planta Daninha, v. 25, n. 2, p. 351-358, 2007. 
MARTINS, D. et al. Efeito do período de exposição a concentrações de diquat no controle de plantas de Egeria densa, Egeria najas e Ceratophyllum dermersum.

Planta Daninha, v. 26, n. 4, p. 865-874, 2008.

MUSTAFA, A. L. et al. A experiência da CESP no manejo e controle de macrófitas no reservatório da UHE Souza Dias (Jupiá). Ação Amb., v. 13, n. 43, p. 17-26, 2010.

NASCIMENTO, P. R. F et al. Biomassa de Egeria densa nos reservatórios da hidroelétrica de Paulo Afonso-Bahia. Planta Daninha, v. 26, n. 3, p. 481-486, 1998.

NETHERLAND, M. et al. Differential response of hydrilla to fluridone. In: AQUATIC PLANT MANAGEMENT SOCIETY, FORTIETH ANNUAL MEETING, 24., San Diego, 2000. Proceedings... San Diego: 2000. p. 24.

PAUL, E. A. et al. The toxicity of diquat, endothal and fluridone to the early life stage of fish. J. Freshwater Ecol., v. 9, n. 3, p. 229-239, 1994.
PITELLI, R. L. C. M. et al. Dinâmica da comunidade de macrófitas aquáticas no reservatório de Santana, RJ. Planta Daninha, v. 26, n. 3, p. 473-480, 2008.

RAND, G. M.; PETROCELLI, S. R. Fundaments of aquatic toxicology. Washington: Hemisphere, 1985. $665 \mathrm{p}$.

SAS Institute. Statistical Analyses System. Cary: 1998.

VELINI, E. D. Desenvolvimento de técnicas e equipamentos para o monitoramento e controle de plantas aquáticas, Botucatu, 2005. 238 f. Dissertação (Livre-Docente) - Universidade Estadual Paulista, Botucatu, 2005.

ZUCKER, E. Standard evaluation procedure. Acute toxicity test for freshwater fish. USEPA publication 540/985-006. Washington, D.C.: 1985. Disponível em: <www.epa.gov>. Acesso em: 18 mar. 2008. 